\title{
Preditores da autoavaliação da Saúde Geral em docentes de Educação a Distância
}

\section{(Predictors of self-assessment of general health in teachers working in distance education)}

\author{
Daniele Kruel Goebel \\ Mary Sandra Carlotto \\ Universidade do Vale do Rio dos Sinos, Unisinos (Brasil)
}

DOI: $\underline{\text { http://dx.doi.org/10.5944/ried.22.1.21885 }}$

\section{Como referenciar este artigo:}

Kruel Goebel, D., y Carlotto, M. S. (2019). Preditores da autoavaliação da Saúde Geral em docentes de Educação a Distância RIED. Revista Iberoamericana de Educación a Distancia, 22(1), pp. 309-344. doi: http://dx.doi.org/10.5944/ ried.22.1.21885

\section{Resumo}

A percepção de saúde abrange tanto questões físicas quanto emocionais, além de aspectos do bem-estar e da satisfação com a própria vida, assim como o seu nível está associado à adoção de comportamentos de saúde. Este artigo tem como objetivo avaliar o poder preditivo dos estressores ocupacionais e psicossociais para a autoavaliação da saúde geral em docentes de EaD e verificar se existe diferença na predição entre professores e tutores. A amostra do tipo não probabilística constituiu-se de 158 professores e 152 tutores de Educação a distância. Foi utilizado um questionário para obter dados sobre autoavaliação em saúde geral, avaliação de estressores ocupacionais e uma escala de avaliação de Estressores Psicossociais no Contexto Laboral. Os resultados, obtidos por meio da análise de regressão linear múltipla, indicaram como variáveis preditoras, nos professores, a carga horária de trabalho, forma como o trabalho é organizado e pressão do grau de responsabilidade e, nos tutores, conciliar trabalho-família, sobrecarga de papéis e relação com o superior imediato. Os resultados indicam especificidades nas variáveis explicativas no que tange à autoavaliação de tutores e professores.

Palavras-chave: saúde geral; estressores ocupacionais; professores; tutores; EaD; saúde do trabalhador. 


\begin{abstract}
The perception of health covers fisical and emotional points it also covers well-being and satisfaction with its own life, as well as its level is related to the adoption of a healthy behavior.This study aims to evaluate the predictive power of occupational and psychosocial stressors for the self-assessment of general health in teachers working in distance education and to verify if there are differences in the predictions between teachers and tutors. The nonprobalistic sample consisted of 158 teachers and 152 tutors working in distance education. A questionnaire was used to obtain data on self-assessment of general health, evaluation of occupational stressors. A scale for assessing psychosocial stressors in the work context was also used. The results, obtained through multiple linear regression analysis, indicated the following burnout predictive variables: in teachers, their work load, the way work is organized and the pressure of the level of responsibility; in tutors, the work-family balance, the overload of roles and the relationship with the immediate supervisor. The results indicate specificities in the explanatory variables regarding the self-assessment of tutors and teachers.
\end{abstract}

Keywords: general health; occupational stressors; teachers; tutors; distance education; worker's health.

Otrabalho e as organizações têm apresentado mudanças significativas nas últimas décadas. As organizações têm sido mais produtivas e competitivas, demandando aos trabalhadores mais capacidades físicas, mentais, cognitivas e emocionais. Porém, mesmo com todas as mudanças significativas do trabalho, esse deve ser saudável, contribuir para o desenvolvimento do trabalhador (Pérez, 2017). A saúde é um estado de completo bem-estar físico, mental e social e não somente ausência de doenças e enfermidades, sendo tal aspecto um dos direitos fundamentais dos indivíduos sem distinção de raça, religião, crença política ou condição econômica ou social (World Health Organization [WHO], 2014).

A operacionalização do conceito de saúde pode ser medida por informações médicas baseadas em sinais e sintomas patológicos, exames diagnósticos e por registros médicos. Outra forma de medida pode ser realizada por avaliações feitas pelos próprios indivíduos, por meio de diversos indicadores sobre seu estado de saúde (Pavão, Werneck, \& Campos, 2013). Para avaliar a saúde são utilizados um conjunto de dados e instrumentos que apontam informações sobre as diferentes dimensões dessa, conhecidos como indicadores de saúde (Abellán et al., 2016).

A percepção de saúde depende do conhecimento em relação à saúde que cada indivíduo apresenta (Pavão et al., 2013; Prass \& Oliveira, 2015). E essa pode ser entendida no sentido mais reducionista, ou seja, considerando, apenas, as questões biológicas, ou ampliada, então, considerando os vários fatores que influenciam sua percepção de saúde (Prass \& Oliveira, 2015). A percepção de saúde abrange tanto componentes físicos quanto emocionais (Abellán et al., 2016; Pavão et al., 2013), além de aspectos do bem-estar e da satisfação com a própria vida (Pavão et al., 2013). 
A autoavaliação da saúde é fundamental, na medida em que a percepção do seu nível está associada à adoção de comportamentos de saúde, já havendo evidências de que a prevalência de comportamentos de risco é maior entre aqueles que possuem uma percepção negativa do seu estado de saúde (Johansson \& Sundquist, 1999; Segovia, Bartlett, \& Edwards, 1989). Em geral, há evidências de que os estilos de vida condicionam um nível mais alto de saúde (Bryant, Bonevski, Paul, \& Lecathelinais, 2013). A autoavaliação em saúde vem sendo utilizada nos estudos epidemiológicos pela sua validade, estabelecida por suas relações com as condições clínicas e com o maior risco de morbilidade e mortalidade subsequente (Szwarcwald, Souza-Júnior, Esteves, Damacena, \& Viacava, 2005). De acordo com os autores, a percepção precária da própria saúde pode ser vista como resultado do sofrimento ou desconforto na interação com fatores sociais, culturais, psicológicos e ambientais. Essa tem sido uma das medidas mais empregadas em inquéritos populacionais, recomendada pela Organização Mundial de Saúde (OMS) para monitoramento do estado de saúde (Petarli, Salaroli, Bissoli, \& Zandonade, 2015).

Revisão sistemática da literatura internacional, realizada por DeSalvo, Bloser, Reynolds, He e Muntner (2006), verificou que as pessoas com menor autoavaliação em saúde apresentavam um risco de mortalidade duas vezes maior quando comparadas com as de maior autoavaliação. No Brasil, investigação de base populacional, realizada por Peres et al. (2010), em uma cidade do sul, com adultos entre 20 a 59 anos, identificou que o número de morbidade se associou fortemente à autoavaliação de saúde negativa.

Especificamente em trabalhadores, a percepção de estar exposto a níveis elevados de estresse relacionou-se à maior prevalência de percepção negativa de saúde (Barros \& Nahas, 2001; Filha, Costa, \& Guilam, 2013). Altas exigências e baixo controle do trabalho, sentimentos de falta de reconhecimento no trabalho e desequilíbrio entre vida pessoal e profissional foram associados com menor autoavaliação da saúde (Milner et al., 2017). Em professores, estudo realizado por Li et al. (2016) revelou que o papel dos estressores foi identificado nos resultados e mostrou que os participantes que possuíam melhor capacidade de gerenciamento dos estressores, também, tinham melhor autoavaliação em saúde.

Os fatores psicossociais do trabalho referem-se às interações do meio ambiente e condições de trabalho, condições organizacionais, funções e conteúdo do trabalho, esforços, características individuais e familiares dos trabalhadores (Organização Internacional do Trabalho [OIT], 1984). Os riscos psicossociais surgem na interação do trabalho, ambiente, condições da organização e satisfação do trabalhador, incluindo, ainda, as suas necessidades e capacidades, sua cultura e situação pessoal fora do ambiente laboral (Chagas, 2015). Por sua vez, podem ser fontes de estresse, e este exerce influência importante sobre o desenvolvimento de desconfortos físicos e psicológicos no ambiente de trabalho (Taylor, Green, \& MPhil, 2015).

Os estressores psicossociais exercem impacto na saúde física e mental mediante processos psicológicos decorrentes da relação do indivíduo com seu contexto de 
trabalho (Ganster \& Rosen, 2013). Os fatores de risco psicossocial mostram-se como um desequilíbrio individual e organizacional cuja permanência, associada à falta de estratégias de controle e prevenção, poderá colocar em risco a saúde dos trabalhadores (Chagas, 2015).

A modalidade de ensino de educação a distância $(\mathrm{EaD})$ vem consolidando-se no mundo em diversos níveis de ensino: técnico, graduação, pós-graduação, bem como em cursos livres ou de extensão (Alves, 2014). Essa modalidade tem apresentado um crescimento significativo, tanto pela procura pelos estudantes quanto ao aumento do número de instituições que oferecem essa modalidade. Os recursos tecnológicos, as propostas metodológicas favoreceram a escolha por essa forma de ensino e aprendizagem.

A principal forma de trabalho dessa modalidade é o uso do Ambiente Virtual de Aprendizagem (AVA), assim, nessa modalidade, o aluno e o professor podem estar em espaços geográficos diferentes e conectados em tempos distintos (Nahón, 2017). Os professores que ministram aulas na $\mathrm{EaD}$ selecionam e preparam o conteúdo curricular articulado a procedimentos e atividades pedagógicas; definem o referencial teórico utilizado nas aulas, a bibliografia, gravam videoaulas e elaboram o material didático para programas a distância; realizam a gestão acadêmica do processo de ensino e aprendizagem, como orientar, acompanhar e avaliar os alunos e avaliarse continuamente como docente participante de um projeto de ensino superior a distância (MEC, 2007). Fornecem feedback (Raffo, Brinthaupt, Gardner, \& Fisher, 2015), gerenciam e monitoram o aprendizado, estimulam a motivação e coesão dos alunos.

O professor de EaD, de acordo com Freitas e Franco (2014), geralmente, não estabelece relação direta com os alunos, mas por meio dos tutores e do material didático impresso, de áudio e/ou digital. Entre suas atribuições, deve identificar necessidades para o planejamento e acompanhamento das aulas em equipe, tutores e profissionais da tecnologia da informação. É o responsável pelo acompanhamento do tutor e por disponibilizar atividades complementares visando preencher lacunas e sanar dificuldades não previstas no planejamento inicial das atividades acadêmicas.

Os tutores da EaD esclarecem dúvidas a partir de fóruns de discussão pela internet, e-mail e realizam webconferências. São responsáveis, também, por promover espaços para diálogos e construção do conhecimento, selecionar material de apoio e sustentação teórica aos conteúdos, bem como, frequentemente, ajudam o professor na preparação das avaliações e participam ativamente da prática pedagógica (MEC, 2007). O tutor trabalha com os alunos, assim, contribuindo para sua aprendizagem com mensagens, recados e orientações por meio do ambiente virtual de aprendizagem (AVA) (Silveira, Souza, \& Silva, 2014). É responsável pelo acompanhamento, pela interação com os alunos, assim, visando garantir o melhor desempenho desses nas atividades propostas (Alves, 2014; Leão, 2014), pela correção de atividades (Guimarães, 2015; Leite, 2015; MEC, 2007) sendo mediador da aprendizagem (Guimarães, 2015). Envolvido nesse processo de aprendizagem, 
também, existe a responsabilidade e comprometimento do aluno (Durán, EstayNiculcar, \& Álvarez 2015) que deve assumir, por sua vez, uma postura mais ativa na sua aprendizagem (Silva, Falcão, Torres, \& Caraballo, 2017).

Pesquisas realizadas com professores e tutores de EaD mostraram que eles apresentam estressores em comum: como a elevada carga horária de trabalho (Leão, 2014; Leite, 2015; Wingo, Ivankova, \& Moss, 2017), o número elevado de alunos (Hackmayer \& Bohadana, 2014; Leite, 2015) o conflito trabalho/lazer (Leão, 2014; Leite, 2015). A literatura relata, ainda, os estressores que os diferenciam: em tutores, a sobrecarga de papéis (Guimarães, 2015; Hackmayer \& Bohadana, 2014; Leite, 2015), as cobranças por parte dos superiores (Leão, 2014). Em professores, o conflito trabalho-família (Luz \& Neto, 2016) e o número elevado de turmas (Leite, 2015).

Assim, considerando as especificidades e os estressores de acordo com o cargo, o presente estudo de delineamento observacional, analítico e transversal teve como objetivo avaliar o poder preditivo dos estressores ocupacionais e psicossociais para a autoavaliação da saúde geral em docentes de $\mathrm{EaD}$ e verificar se existe diferença na predição entre professores e tutores.

\section{MÉTODO}

\section{Participantes}

A amostra não probabilística foi composta por 158 professores e 152 tutores de $\mathrm{EaD}$, de ensino superior de universidades públicas e privadas de todo o Brasil, considerando os seguintes critérios de inclusão: estar em atividade há mais de 1 ano como docente de $\mathrm{EaD}$.

No que se refere aos tutores, $67,1 \%$ eram do sexo feminino, $67,1 \%$ possuíam companheiro e $49 \%$ tinham filhos. A idade variou entre 24 a 64 anos $(\mathrm{M}=39,3$; $\mathrm{DP}=9,11)$. Quanto à formação, a maior parte possuía especialização (48\%), seguida de mestrado (42,1\%), doutorado $(5,9 \%)$ e graduação $(3,9 \%)$. Quanto à remuneração, a maioria pertencia a classe econômica C ( $\mathrm{R} \$ 1.734,00$ a 7.475,00) $(67,1 \%)$, seguida pelas classes $\mathrm{D}(\mathrm{R} \$ 1.085,00$ a $1.734,00)(11,2 \%)$, A (acima de $\mathrm{R} \$ 9.745,00)(9,9 \%)$, $\mathrm{E}$ (menos de $\mathrm{R} \$ 1.085,00)(7,9 \%)$ e $\mathrm{B}(\mathrm{R} \$ 7.475,00$ a $9.745,00)(3,9 \%)$, de acordo com a classificação da Fundação Getúlio Vargas-Brasil/Centro de Políticas Sociais elaborada por Neri (2012).

Quanto ao local de trabalho, a maioria indicou que trabalhava em universidade privada (79,6\%). O tempo médio de atuação dos profissionais em docência era entre 1 a 15 anos em $\mathrm{EaD}(\mathrm{M}=5,84 ; \mathrm{DP}=3,52)$. Verificou-se, também, que os tutores possuíam carga horária contratual que variava de $1 \mathrm{~h}$ a $60 \mathrm{~h}$ semanais em $\mathrm{EaD}$ $(\mathrm{M}=21,3 ; \mathrm{DP}=12,43)$ e atendiam entre 10 a 3.000 alunos $(\mathrm{M}=401,73$; $\mathrm{DP}=570,73)$.

Os professores, na sua maioria, eram do sexo feminino (59,5\%), com companheiro (67,8\%) e com filhos (51\%). A idade variou entre 27 a 71 anos $(M=44,3$; $\mathrm{DP}=9,63)$. Quanto à formação, a maioria possuía mestrado $(51,9 \%)$, seguido de 
doutorado (27,2\%), especialização $(17,1 \%)$ e graduação $(3,8 \%)$. No que diz respeito à remuneração, o maior percentual de professores situa-se na classe econômica $\mathrm{C}(\mathrm{R} \$ 1.734,00$ a $7.475,00)(45,6 \%)$, seguida pelas classes A (acima de 9.745,00) (32,9\%), B (R \$7.475,00 a 9.745,00) (20,3\%), D (R $\$ 1.085,00$ a $1.734,00)(0,6 \%)$ e E (menos de $\mathrm{R} \$ 1.085,00)(0,6 \%)$. Quanto ao local de trabalho, a maioria trabalhava em universidade privada (86,7\%). O tempo médio de atuação dos profissionais em docência variou entre 1 a 23 anos em $\operatorname{EaD}(M=6,41 ; \mathrm{DP}=3,84)$. Verificou-se, também, que os professores apresentavam carga horária contratual que variava de $1 \mathrm{~h}$ a $44 \mathrm{~h}$ semanais em $\mathrm{EaD}(\mathrm{M}=16,36 ; \mathrm{DP}=12,58)$. Os professores atendiam entre 10 a 2.500 alunos $(\mathrm{M}=344,78 ; \mathrm{DP}=492,93)$.

\section{Instrumentos}

Para atingir os objetivos propostos neste estudo, utilizou-se quatro instrumentos autoaplicáveis:

1. Questionário de dados sociodemográficos (situação conjugal, sexo, idade, filhos, remuneração) e laborais (cargo, formação, local de trabalho, tempo de experiência profissional no EaD, carga horária, total de alunos).

2. A autoavaliação de saúde geral foi avaliada pela questão: "Como você percebe sua saúde em geral?”, cuja escala de medida variou de 1 (ruim) a 10 (ótima).

3. Escala para Avaliação de Estressores Psicossociais no Contexto Laboral (Ferreira et al., 2015), constituída por 35 itens, distribuídos em sete dimensões: conflito e ambiguidade de papéis; ( 5 itens, $\boldsymbol{\alpha}=0,80$, ex.: Não saber exatamente o que esperam de mim em meu trabalho); sobrecarga de papéis (6 itens, $\alpha=0,75$, ex.: Ter um ritmo acelerado de trabalho); falta de suporte social (6 itens, $\alpha=$ 0,82, ex.: Não receber ajuda de meus superiores quando tenho algum problema pessoal); insegurança na carreira ( 4 itens, $\alpha=0,72$, ex.: Conviver com rumores sobre cortes de pessoal); falta de autonomia ( 5 itens, $\alpha=0,74$, ex.: Não poder decidir sobre quando tirar férias); conflito trabalho-família (5 itens, $\alpha=0,75$, ex.: Não dispor de tempo para minha vida pessoal em função do trabalho); pressão do grau de responsabilidade ( 4 itens, $\alpha=0,77$, ex.: Saber que meus erros podem prejudicar a mim mesmo). Os itens foram avaliados com uma escala de frequência de 6 pontos (1 "nunca" a 6 "sempre me afeta").

4. Questionário de estressores ocupacionais EaD foi elaborado para o presente estudo com base na literatura sobre estressores em professores em geral (Carlotto \& Palazzo, 2006) e de EaD (Raffo, Brinthaupt, Gardner, \& Fisher, 2015). Essa possui 12 itens (carga horária, horário de trabalho, alunos por turma, carga de trabalho diária, diversidade de atividades diárias, administração dos diversos sistemas, relação com o superior imediato, relação com os alunos, organização do trabalho, necessidade de atualização, conciliar trabalho-família, conciliar trabalho-lazer), que foram avaliados por meio de uma escala de cinco pontos (o "nada estressante" a 4 "altamente estressante"). 


\section{Procedimentos de coleta de dados}

A coleta de dados foi realizada de forma online por meio de formulário eletrônico, via redes sociais e e-mails de contato da pesquisadora e dos membros do grupo de pesquisa em Psicologia da Saúde Ocupacional/UNISINOS. O período de realização da coleta ocorreu entre os meses de abril a agosto de 2017.O estudo foi aprovado pelo Comitê de Ética em Pesquisa da Universidade Vale do Rio Sinos (UNISINOS), sob o número CAAE: 62592316.1.0000.5344. $\mathrm{O}$ acesso à pesquisa somente era possível após o aceite do Termo de Consentimento Livre e Esclarecido (TCLE).

\section{Procedimentos de análise de dados}

O banco de dados foi analisado por meio do SPSS 20 (Statistical Package for the Social Sciences). Primeiramente, foram realizadas análises descritivas de caráter exploratório com o intuito de avaliar a distribuição dos itens e os possíveis erros de digitação. Para a verificação de diferenças de variáveis sociodemográficas e laborais entre os grupos de professores $(\mathrm{P})$ e tutores $(\mathrm{T})$ foram utilizados o teste QuiQuadrado, para variáveis qualitativas, e o t de student, para variáveis quantitativas.

Para identificar os preditores foi realizada análise de regressão linear múltipla, método Stepwise, após realização de seus pressupostos de acordo com Field (2009). Foi verificada ausência de multicolinearidade, pois todos os valores das correlações ficaram abaixo de 0,678 (P) e 0,709 (T), os valores de Variance Inflation Factor (VIF) situaram-se abaixo de 4, 1,248 (P) e 1,291 (T), e os valores de tolerância foram inferiores a 1, 0,801 (P) e 0,775 (T). A análise do coeficiente de Durbin-Watson identificou valores próximo a 2, 1,688 (P) e 1,948 (T), indicando a independência da distribuição e a não correlação dos resíduos. A distância de Cook apresentou valores de 0,008 (P) e 0,009 (T), inferiores a 1, indicando não existir preditores atípicos e um adequado ajuste dos modelos.

O modelo proposto estabelece a saúde geral como variável dependente (VD) e os estressores laborais e psicossociais como independentes (VIs). A magnitude do efeito (effect size) foi avaliada pelos coeficientes de regressão padronizados calculados para cada modelo final, de acordo com Field (2009). O tratamento dos dados adotou um

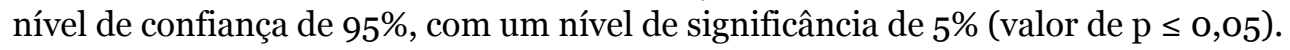

\section{RESULTADOS}

Os grupos de professores (P) e tutores (T) apresentaram diferença significativa, avaliada pelo teste $t$ de student, nas variáveis idade ( $p=0,000 ; t=-4,702)$, tempo de atuação profissional ( $\mathrm{p}=0,000 ; t=-7,538)$, tempo de atuação de $\operatorname{Ead}(\mathrm{p}=0,004$; $t=-2,919)$, carga horária semanal na graduação presencial $(\mathrm{p}=0,000 ; t=-7,538)$, tendo os professores apresentado maiores médias. Quanto às variáveis remuneração 
$(\mathrm{p}=0,000 ; t=-8,317)$, formação $(\mathrm{p}=0,000 ; t=-6,377)$, avaliadas por meio do teste qui-quadrado, os professores apresentaram percentuais mais elevados. As variáveis nas quais o tutor apresentou maior média foi na carga horária semanal contratual na $\mathrm{EaD}(\mathrm{p}=0,001 ; t=3,372)$ e no número de disciplinas de $\mathrm{EaD}(\mathrm{p}=0,000 ; t=4,461)$. Quanto à autoavaliação geral de saúde, não foi identificada diferença significativa entre os grupos $(\mathrm{p}=0,576, t=0,560)$.

Resultados obtidos mediante a análise de regressão linear múltipla (Tabela 1) revelaram um modelo preditivo para os tutores constituído por 3 variáveis que, conjuntamente, explicaram 26,8\% da variância desta dimensão, sendo conciliar trabalho-família a variável de maior poder explicativo (19,1\%), seguida pela sobrecarga de papéis e a relação com o superior imediato. $\mathrm{O}$ conjunto de variáveis revelou que, à medida que aumenta a percepção dos estressores conciliar o trabalho com a família e relação com o superior imediato e a sobrecarga de papéis, menor é a autoavaliação de saúde do tutor.

Para os professores, o modelo preditor foi constituído pelas variáveis: carga horária de trabalho, forma com que o trabalho é organizado e pressão por responsabilidades que explicaram conjuntamente $23,3 \%$ da variância. $\mathrm{O}$ resultado indica que quanto maior a carga horária de trabalho, a pressão por responsabilidades e a percepção de que a forma que o trabalho deste é organizado é um estressor, menor é a autoavaliação da sua saúde. Destaca-se que a variável com maior poder explicativo foi a carga horária de trabalho (17,9\%).

Os resultados indicam uma magnitude do efeito média $\left(\mathrm{R}^{2}=0,233\right.$ e 0,268$)$ de acordo com os parâmetros recomendados por Field (2009). Nesse sentido, indica-se que as relações identificadas, possivelmente, também, possam ser encontradas na população-alvo de docentes de EaD.

Tabela 1. Modelo da análise de regressão linear múltipla da Saúde Geral (VD) e estressores ocupacionais e psicossociais (VI)

\begin{tabular}{|c|c|c|c|c|c|c|c|c|}
\hline Cargo & Variáveis & $\mathrm{R}$ & $\mathrm{R}^{2}$ & B & SE & $\beta$ & $t$ & $p$ \\
\hline \multirow{4}{*}{ Tutor } & $\begin{array}{l}\text { Saúde Geral } \\
\text { Conciliar trabalho } \\
\text { familia }\end{array}$ & 0,437 & 0,191 & $-0,375$ & 0,119 & $-0,264$ & $-3,157$ & $0,002^{* * *}$ \\
\hline & Sobrecarga de papéis & 0,492 & 0,243 & $-0,315$ & 0,133 & $-0,204$ & $-2,363$ & $0,019^{*}$ \\
\hline & $\begin{array}{l}\text { Relação superior } \\
\text { imediato }\end{array}$ & 0,518 & 0,268 & $-0,278$ & 0,123 & $-0,181$ & $-2,264$ & $0,025^{*}$ \\
\hline & Modelo F & & & & 17,946 & & & \\
\hline
\end{tabular}




\begin{tabular}{|c|l|c|c|c|c|c|c|c|}
\hline Cargo & \multicolumn{1}{|c|}{ Variáveis } & $\mathrm{R}$ & $\mathrm{R}^{2}$ & $\mathrm{~B}$ & $\mathrm{SE}$ & $\beta$ & $t$ & $p$ \\
\hline \multirow{2}{*}{ Professor } & $\begin{array}{l}\text { Carga horária de } \\
\text { trabalho }\end{array}$ & 0,423 & 0,179 & $-0,375$ & 0,147 & $-0,241$ & $-2,550$ & $0,012^{*}$ \\
\cline { 2 - 9 } & $\begin{array}{l}\text { Organização do } \\
\text { trabalho }\end{array}$ & 0,462 & 0,214 & $-0,398$ & 0,177 & $-0,216$ & $-2,249$ & $0,026^{*}$ \\
\cline { 2 - 9 } & $\begin{array}{l}\text { Pressão por } \\
\text { Responsabilidades }\end{array}$ & 0,483 & 0,233 & $-0,202$ & 0,102 & $-0,147$ & $-1,978$ & $0,050^{*}$ \\
\hline & Modelo F & \multicolumn{7}{|c|}{${ }^{*} p<0.05 ;{ }^{* *} p<0.01 ;$} \\
\hline
\end{tabular}

\section{DISCUSSÃO}

O presente estudo buscou avaliar o poder preditivo dos estressores ocupacionais e psicossociais para a autoavaliação da saúde geral em docentes de $\mathrm{EaD}$ e verificar se existe diferença na predição entre professores e tutores. Os resultados indicam diferenças nas variáveis explicativas da saúde geral entre os grupos, em tutores, foi predita pelo estressor psicossocial sobrecarga de papéis e pelos estressores ocupacionais conciliar trabalho/família, relação com o superior imediato; e, em professores, pelo estressor psicossocial pressão pelo grau de responsabilidades e pelos estressores ocupacionais carga horária de trabalho, forma de organização do trabalho.

O modelopreditivo em tutores indicou queo aumento da percepção dos estressores conciliar o trabalho com a família e relação com o superior imediato e a sobrecarga de papéis diminui a autoavaliação de saúde. No tocante à sobrecarga de papéis, o tutor de EaD tem diversas atividades para realizar, interagir em fóruns de conteúdo, corrigir atividades, dar feedbacks, realizar chats, webconferências, responder a mensagens, sanar dúvidas, porém, muitas vezes, o tempo do contrato do docente não é suficiente para realizar todas as atividades (Guimarães, 2015; Hackmayer \& Bohadana, 2014; Leite, 2015), assim como conciliar o tempo do trabalho com a família (Luz \& Neto 2016), pois, nessa modalidade de ensino, o docente trabalha em casa ou em qualquer outro lugar (Durán, Estay-Niculcar, \& Álvarez, 2015), desde que tenha a ferramenta de trabalho, internet e computador, expandindo o tempo do trabalho para dar conta de suas responsabilidades, possivelmente, invadindo o espaço de suas outras atividades (Leão, 2014). Ou seja, a percepção desses estressores pode fazer com que os tutores avaliem sua saúde negativamente.

No que se refere ao superior imediato, esse resultado pode ser explicado pela posição hierárquica do tutor, pois suas atividades estão sob a responsabilidade, também, do professor (Silva et al., 2017). Nessa relação funcional, segundo Leite (2015), há divergências sobre as atribuições e dificuldade em separar os papéis profissionais que provocam questionamentos que, não raras vezes, configuram-se como um estressor ocupacional. As relações são fatores de impacto na educação 
quando somadas a um trabalho que prevê um exercício de planejar e avaliar conjuntamente com pares, conforme vemos na $\mathrm{EaD}$ (Abreu, Coelho, \& Ribeiro, 2016). Existem, ainda, aqueles tutores que recebem mais liberdade para realizar o seu trabalho e aqueles que são monitorados pelo professor (Leite, 2015). Por essas questões, pode-se supor que essa relação, quando tensionada e configurada como um estressor, faça com que o tutor sinta impacto na sua saúde e tenha uma percepção negativa da saúde.

O modelo preditivo dos professores evidencia que o aumento da percepção dos estressores carga horária de trabalho, forma como o trabalho é organizado e pressão pelo grau de responsabilidade diminui a autoavaliação de saúde. O professor, nesse contexto, além das atividades com os alunos, orienta os tutores quanto às ações didático-pedagógicas, bem como à revisão das comunidades virtuais, à atualização de conteúdos, cronogramas e instrumentos de avaliação com uma carga horária, geralmente, insuficiente (Leite, 2015). Ou seja, a carga horária de trabalho contratual não é suficiente para que ele possa realizar todas as suas atribuições, consequentemente, esse estressor faz com que isso possa diminuir a autoavaliação em saúde.

Quanto à forma como o trabalho é organizado na $\mathrm{EaD}$, as aulas são realizadas pela plataforma on-line (Durán, Estay-Niculcar, \& Álvarez, 2015) e contam com recursos de fóruns, email, webconferências. A comunicação com o aluno dá-se pelo uso da tecnologia, essa é a ferramenta de trabalho. Além disso, esse formato produz novas exigências aos docentes, pois não há um tempo e um local predeterminados para ensinar (Silva et al., 2017). Na EaD, o docente deve programar o conteúdo e as aulas com antecedência, tendo em vista que essas aulas precisam ser disponibilizadas na plataforma on-line em tempo hábil para que o aluno possa realizar a atividade proposta, diferente do modo presencial, em que o professor já disponibiliza na hora o material a ser utilizado. O professor deve conhecer e saber utilizar a plataforma de aprendizagem para que possa realizar as atribuições de sua função.

Quanto à pressão do grau de responsabilidade, esse preditor é em relação ao grau de responsabilidade com as pessoas e os equipamentos que o empregado possui no desempenho de suas funções (Ferreira et al., 2014). Suas contribuições envolvem a interação com os alunos, bem como, avaliá-los e os motivá-los para a realização das atividades, orientação aos tutores quanto às ações didático-pedagógicas, também, a construção e atualização de conteúdos, cronogramas e acompanhamento das disciplinas, definição de referencial bibliográfico e instrumentos de avaliação (Filho, Amaral, \& Schimiguel, 2015; Leite, 2015; MEC, 2007). Pode-se inferir que, no caso de professores, estes possuam tanto responsabilidades pedagógicas quanto em relação ao aluno e ao tutor. Atuar como professor exige que se tenha muitas responsabilidades e atribuições, e isso pode estar, de alguma forma, impactando na sua percepção de saúde. 


\section{CONSIDERAÇÕES FINAIS}

Os resultados deste estudo identificaram modelos preditivos diferenciados para professores e tutores quanto à autoavaliação de saúde geral. As diferenças nos modelos preditivos podem estar relacionadas às características sociodemográficas $\mathrm{e}$ laborais dos professores e tutores. Os professores apresentavam idade mais elevada, maior remuneração, formação e tempo de atuação profissional tanto no ensino presencial quanto na EaD. Já os tutores indicavam uma carga horária semanal contratual maior na $\mathrm{EaD}$ e maior número de disciplinas.

Nesse sentido, as diferenças foram, predominantemente, de caráter funcional, o professor, comumente, além de ministrar aula na EaD, possui uma ampla experiência na modalidade presencial e costuma não estabelecer relação direta com os alunos, mas sim por meio dos tutores. O tutor de $\mathrm{EaD}$ é responsável pela interação com os alunos, medeia os debates, informa os prazos de entregas, auxilia os alunos a familiarizarem-se com o ambiente e com os colegas.

\section{Forças, limitações e sugestões de novos estudos}

É importante destacar duas importantes forças do presente estudo, a primeira diz respeito à média magnitude do efeito encontrada, considerando a diversidade de variáveis que podem explicar a saúde geral na amostra investigada e a segunda refere-se á adequada confiabilidade obtida nas medidas utilizadas e utilização de um instrumento de medida de avaliação geral da saúde consolidado internacionalmente.

Os resultados deste estudo devem ser interpretados considerando algumas limitações como a utilização de medidas de autorrelato e, portanto, podem estar sujeitas à influência do que se espera socialmente como resposta e também em relação a o tipo de amostra não probabilística que não permite a generalização de seus resultados.

Assim, considerando ser este o primeiro estudo nacional de autoavaliação em saúde com docentes EaD, sugere-se sua replicação com amostra nacional aleatória estratificada por regiões, medida que possibilitaria a comparação de resultados e ampliação os achados atuais. Por fim, sugere-se a inclusão de variáveis voltadas para hábitos e estilo de vida a fim de aumentar o poder explicativo da saúde geral.

\section{Implicações para a prática}

Como implicações para a prática, especificamente para os tutores, pode-se pensar em ações voltadas para a promoção da qualidade de vida e do trabalho, principalmente na importância da gestão e equilíbrio do tempo dedicado ao trabalho, lazer e família. É importante, também, investimentos na qualidade do relacionamento entre os tutores e professores, no sentido de maior sensibilização quanto à importância do apoio social e estabelecimento de relações sinérgicas e cooperativas. 
Para os professores, sugere-se que os gestores institucionais realizem uma análise de função e atribuições e sua compatibilidade com a carga horária contratual do professor. Este redimensionamento proporcionaria melhorias na qualidade e organização do trabalho e a diminuição da pressão e responsabilização por resultados percebidas pelo professor.

Por fim, as instituições de ensino devem atuar preventivamente em possíveis problemas que possam ocorrer relacionados ao trabalho nessa modalidade de ensino, como, por exemplo, conhecer as consequências do trabalho na vida social e na saúde do docente, tanto física quanto psíquica.

\section{REFERÊNCIAS}

Abellán, B. G., Hidalgo, L. T. J., Sotos, R. J., López, T. L., \& Jiménez, V. C. (2016). Alimentación saludable y autopercepción de salud. Aten Primaria, 48(8), 535542. doi: https://doi.org/10.1016/j. aprim.2015.12.001

Abreu, M., Coelho, M. D., \& Ribeiro, S. J. (2016). Percepção de professores universitários sobre as repercussões do seu trabalho na própria saúde. Revista Brasileira De Pós-Graduação, 13(31), 465-486. doi: https://dx.doi. org/10.21713/2358-2332.2016.v13.1155

Alves, S. M. (2014). A ativid@de de tutores na educação a distância: um olhar ergológico para o prescrito e o vivido nos ambientes virtuais de aprendizagem. Letras de Hoje, 49(3), 336-344. doi: https://dx.doi. org/10.15448/1984-7726.2014.3.15870

Barros, M. V. G. de, \& Nahas, M. V. (2001). Comportamentos de risco, auto-avaliação do nível de saúde e percepção de estresse entre trabalhadores da indústria. Revista de Saúde Pública, 35(6), 554-563. doi: https://dx.doi.org/10.1590/s003489102001000600009

Bryant, J., Bonevski, B., Paul, C. L., \& Lecathelinais, C. L. (2013). A cross-sectional survey of health risk behaviour clusters among a sample of socially disadvantaged Australian welfare recipients. Aust $N Z J$ Public
Health, 37, 118-123. doi: https://doi. org/10.1111/1753-6405.12028

Carlotto, M. S., \& Palazzo, L. S. (2006). Síndrome de burnout e fatores associados: um estudo epidemiológico com professores. Cadernos de Saúde Pública, 22(5), 1017-1026. doi: https://dx.doi. org/10.1590/s0102-311x2006000500014

Chagas, D. (2015). Riscos psicossociais no trabalho: causas e consequências. Revista Infad de Psicologia, 2(1), 439446. doi: https://dx.doi.org/10.1706o/ ijodaep.2015.n1.v2.24

DeSalvo, K. B., Bloser, N., Reynolds, K., He, J., \& Muntner, P. (2006). Mortality prediction with a single general self- rated health question: a meta-analysis. Journal of general internal medicine, 21(3), 267275. doi: https://dx.doi.org/10.1111/ j.1525-1497.2005.00291.x

Durán, R., Estay-Niculcar, C., \& Álvarez, H. (2015). Adopción de buenas prácticas en la educación virtual en la educación superior. Aula Abierta, 43, 77-86. doi: http://dx.doi.org/10.1016/j. aula.2015.01.001 0210-2773

Ferreira, M. C., Milfont, T. L., Silva, A. P. C., Fernandes, H. A., Almeida, S. P., \& Mendonça, H. (2015). Escala para avaliação de estressores psicossociais no contexto laboral: construção e evidências de validade. Psicologia: Reflexão e 
Crítica, 28(2), 340-349. doi: https:// dx.doi.org/10.1590/1678-7153.201528214

Field, A. (2009). Descobrindo a estatística usando o SPSS-2. Editora: Bookman.

Filha, M. M. T., Costa, M. A. S., \& Guilam, M. C. R. (2013). Occupational stress and self-rated health among nurses. Revista Latino-Americana de Enfermagem, 21(2), 475-483. doi: https://dx.doi. org/10.1590/s0104-11692013000200002

Filho, G. F. A., Amaral, L. H., \& Schimiguel, J. (2015). A importância do professor na educação a distância. Revista Atlante. Disponível em http://www.eumed.net/ rev/atlante/o4/educacion-distancia.html

Freitas, M. T. M., \& Franco, A. P. (2014). Os desafios de formar-se professor formador e autor na educação a distância. Educar em Revista, 4, 149-172. doi: https:// dx.doi.org/10.1590/0104-4060.38658

Ganster, D. C., \& Rosen, C. C. (2013). Work stress and employee health: a multidisciplinary review. Journal of Management, 39(5), 1085-1122. doi: https://dx.doi. org/10.1177/0149206313475815

Guimarães, B. V. (2015). Tutoria em educação a distância: papéis, formações e percepções. Dissertação de Mestrado, Unirio, Duque de Caxias, RJ, Brasil.

Hackmayer, M. B., \& Bohadana, E. (2014). Professor ou tutor: uma linha tênue na docência em ead. RIED. Revista Iberoamericana de Educación a Distancia, 17(2), 223-240. doi: https:// dx.doi.org/10.5944/ried.17.2.12685

Johansson, S., \& Sundquist, J. (1999). Change in lifestyle factors and their influence on health status and all-cause mortality. International Journal Epidemiol, 28(6), 1073-1080. doi: https://dx,doi. org/10.1093/ije/28.6.1073

Leão, F. D. L. P. (2014). Relações saúde, trabalho e resiliência do Docente - tutor na Educação a Distância. Dissertação de Mestrado, Universidade de Taubaté, Taubaté, São Paulo, SP, Brasil.
Leite, T. C. (2015). Docência compartilhada e o potencial da formação continuada: rotas possíveis nas viagens pelas práticas docentes na EaD. Dissertação de Mestrado, Universidade do Vale do Rio dos Sinos- Unisinos, São Leopoldo, RS, Brasil.

Li, F., Chen, J., Yu, L., Jing, Y., Jiang, P., Fu, X., Wu, S., Sun, X., Luo, R., Kwan, H., Zhao, X., \& Liu, Y. (2016). The role of stress management in the relationship between purpose in life and self-rated health in teachers: a mediation analysis. International Journal of Environmental Research and Public Health, 13(7), 1-9. doi: https://dx.doi.org/10.3390/ ijerph13070719

Luz, M. A. M., \& Neto, J. L. F. (2016). Processos de trabalho e de subjetivação de professores universitários de cursos de educação à distância. Psicologia Escolar e Educacional, 20(2), 265-274. doi: https://dx.doi.org/10.1590/2175353920150202962

MEC/SEED - Ministério da Educação / Secretaria de Educação a Distância. (2007) Referenciais de qualidade para a educação superior a distância. Brasília. Disponível em http://portal.mec.gov.br/ seed/arquivos/pdf/dec 5622.pdf

Milner, A., Witt, K., Spittal, M. J., Bismark, M., Graham, M., \& La Montagne, A. D. (2017). The relationship between working conditions and self-rated health among medical doctors: evidence from seven waves of the Medicine In Australia Balancing Employment and Life (Mabel) survey. BMC Health Services Research, 17(1), 1-10. doi: https://dx.doi. org/10.1186/s12913-017-2554-Z

Nahón, E. A. (2017). Aportaciones al proceso horizontal de transversalización de la Educación a Distancia en las instituciones de educación superior. Revista de la Educación Superior 46(182), 5769. doi: http://dx.doi.org/10.1016/j. resu.2017.02.003 
Neri, M. C. (2012). De Volta ao País do Futuro: Crise Européia, Projeções e a Nova Classe Média. Rio de Janeiro: FGV/ CPS.

Organização Internacional do Trabalho. (1984). A condição dos professores: recomendação Internacional de 1966, um instrumento para a melhoria da condição dos professores. Genebra: OIT/ Unesco.

Pavão, A. L. B., Werneck, G. L., \& Campos, M. R. (2013). Autoavaliação do estado de saúde e a associação com fatores sociodemográficos, hábitos de vida e morbidade na população: um inquérito nacional. Cadernos De Saúde Pública, 29(4), 723-734. doi: https://dx.doi. org/10.1590/s0102-311x2013000400010

Pérez, J. P. (2017). Salud ocupacional y psicología: ¿Quo vadis? Revista Cubana de Salud y Trabajo, 18(1), 67-73. Disponível em http://www.bvs.sld.cu/ revistas/rst/vol18 11 17/rsto9117.htm

Peres, M. A., Masiero, A. V., Longo, G. Z., Rocha, G. C., Matos, I. B., Najnie, K., Oliveira, C. M., Arruda, M. P., \& Peres, K. G. (2010). Auto-avaliação da saúde em adultos no Sul do Brasil. Revista de Saúde Pública, 44(5), 901-911. doi: https://dx.doi.org/10.1590/s0034$\underline{89102010000500016}$

Petarli, G. B., Salaroli, L. B., Bissoli, N. S., \& Zandonade, E. (2015). Autoavaliação do estado de saúde e fatores associados: um estudo em trabalhadores bancários. Cadernos de Saúde Pública, 31(4), 787-799. doi: https://dx.doi. org/10.1590/0102-311x00083114

Prass, P. B. B., \& Oliveira, W. S. (2015). A interface da autoavaliação em saúde dos professores e seu preparo para abordagem do tema na escola. Revista Brasileira de Ciências da Saúde, 19(2), 45-52. doi: https://dx.doi.org/10.4034/rbcs.2015.19. $\underline{\mathbf{s 2 . 0 7}}$

Raffo, D. M., Brinthaupt, T. M., Gardner, J. G., \& Fisher, L. S. (2015). Balancing online teaching activities: strategies for optimizing efficiency and effectiveness. Online Journal of Distance Learning Administration,18(1). Disponível em http://www.westga.edu/ distance/ojdla/ spring181/raffo brinthaupt gardner fisher181.html

Segovia, J., Bartlett, R. F., \& Edwards, A. C. (1989). The association between selfassessed health status and individual health practices. Canadian Journal of Public Health, 8o(1), 32-37. Disponível em http://europepmc.org/abstract/ med/2702542

Silva, A. K. L., Falcão, J. T. R., Torres, C. C., \& Caraballo, G. P. (2017). Os impedimentos da atividade de trabalho do professor na EAD. Psicologia: Ciência E Profissão, 37(3), 683-696. doi: https://dx.doi. org/10.1590/1982-3703004162015

Silveira, R. A. M., Souza, M. M. P., \& Silva, W. V. K. M. (2014). O papel do tutor como mediador da aprendizagem na educação a distância. Disponível em http://www. abed.org.br/hotsite/20-ciaed/pt/anais/ pdf/192.pdf

Szwarcwald, C. L., Souza-Júnior, P. R. B., Esteves, M. A. P., Damacena, G. N., \& Viacava, F. (2005). Socio-demographic determinants of self-rated health in Brazil. Cadernos de Saúde Pública, 21(Suppl.1), 54-64. doi: https://dx.doi.org/10.1590/ $\underline{\text { s0102-311X2005000700007 }}$

Taylor, K., Green, N., \& MPhil. (2015). Psychosocial risk factors: what are they and why are they important? Otago: Wellnomics. Disponível em http://www.workpace.com/ assets/Uploads/WhitePapers/ WellnomicsWhitepaperPsychosocialrisk-factors-What-are-they-and-why-arethey-important.pdf

Wingo, N. P., Ivankova, N. V., \& Moss, J. A. (2017). Faculty Perceptions about Teaching Online: Exploring the Literature Using the Technology Acceptance Model as an Organizing Framework. Online 
Learning, 21(1), 15-35. doi: http://dx.doi. org/10.24059/olj.v21i1.761

World Health Organization (2014). Constitution of the World Health Organization.
Basic Documents. Genebra: WHO. Disponível em http://apps.who.int/iris/bitstr eam/10665/151605/1/9789241650489 eng.pdf

\section{PERFIL ACADÊMICO E PROFISSIONAL DOS AUTORES}

Daniele Kruel Goebel. Mestre em Psicologia Clínica pela Universidade do Vale do Rio dos Sinos. Especialista em RH e Marketing (FAMES). Graduada em Psicologia pelo Centro Universitário Franciscano. É membro do Grupo de Pesquisa em Saúde Ocupacional (GPPSO) Unisinos. Tutora EaD na Universidade do Vale do Rio dos Sinos (UNISINOS).

E-mail: danielekgoebel@gmail.com

Endereço:

Rua Pinheiro Machado, 236

Bairro São José

Canoas - RS, Brasil

CEP: 92420320

Mary Sandra Carlotto. Doutora em Psicologia Social pela Universidade de Santiago de Compostela/Espanha. Graduação em Psicologia pela Universidade Vale do Rio dos Sinos. Membro da equipe de investigação da Unidad de Investigación Psicosocial de la Conducta Organizacional (UNIPSICO) - Universidade de Valencia - Espanha. Membership of European Academy of Occupational Health Psychology. Professora do Curso de Psicologia e do PPG em Psicologia na Universidade do Vale do Rio dos Sinos (UNISINOS).

E-mail: mscarlotto@gmail.com

Endereço:

Universidade do Vale do Rio dos Sinos

Programa de Pós-Graduação em Psicologia- Escola de Saúde - Sala Eo1-118

Av. Unisinos, 950

Bairro: Cristo Rei

São Leopoldo - RS/Brasil

CEP: $93.022-750$

Fecha de recepción del artículo: 14/04/2018

Fecha de aceptación del artículo: 23/04/2018

Fecha de aprobación para maquetación: 06/10/2018 